\title{
Vital dyes in macular hole surgery
}

\author{
IOANA STELLA (PATONI) POPESCU ${ }^{1 *}$, OVIDIU MUȘAT ${ }^{2 *}$, SIMONA STANCA $^{3 *}$, \\ CORINA CRISTINA (COMAN) CERNAT ${ }^{1}$, CRISTINA PATONI ${ }^{4}$, SERBAN NEGRU ${ }^{5}$, \\ ADRIAN TEODORU $^{6}$, HORIA FEIER ${ }^{7}$ and CIPRIAN DANIELESCU ${ }^{8}$
}

\author{
${ }^{1}$ Department of Ophthalmology, 'Victor Babeş' University of Medicine and Pharmacy Timisoara, 300041 Timisoara; \\ ${ }^{2}$ Department of Ophthalmology, 'Dr. Carol Davila' Central Military Emergency University Hospital, 010825 Bucharest; \\ ${ }^{3}$ Department of Pediatrics, 'Carol Davila' University of Medicine and Pharmacy, 050474 Bucharest; \\ ${ }^{4}$ Department of Gastroenterology, 'Dr. Carol Davila' Central Military Emergency University Hospital, 010825 Bucharest; \\ ${ }^{5}$ Department of Oncology, 'Victor Babeş’ University of Medicine and Pharmacy Timisoara, 300239 Timisoara; \\ ${ }^{6}$ Department of Ophthalmology, 'Lucian Blaga' University of Sibiu, 550169 Sibiu; ${ }^{7}$ Department of Cardiovascular Surgery, \\ 'Victor Babeş' University of Medicine and Pharmacy Timisoara, 300041 Timisoara; ${ }^{8}$ Department of Ophthalmology, \\ 'Grigore T. Popa' University of Medicine and Pharmacy, 700115 Iasi, Romania
}

Received November 4, 2020; Accepted December 4, 2020

DOI: $10.3892 / \mathrm{etm} .2021 .9959$

\begin{abstract}
Currently, surgical techniques, such as internal limiting membrane peeling, are used widely for macular holes, macular puckers, epiretinal membranes, diabetic macular edema, retinal detachment, retinal vein occlusions, vitreomacular traction, optic pit maculopathy, and Terson syndrome. This study aimed to highlight any differences regarding visual acuity and ocular tomography coherence changes after staining the internal limiting membrane with dilutions of Brilliant Blue G vs. lutein/zeaxanthin-based dyes. This study involved 30 eyes of 30 patients who had undergone posterior pole vitrectomy for idiopathic stage 4 macular hole. The study lot was divided in two subgroups, 15 eyes colored with Brilliant Blue and the other 15 eyes colored with lutein and zeaxanthin dyes. The association between visual prognosis, ocular tomography coherence changes and intraocular pressure was analyzed. The surgical treatment with required endoillumination levels and a 2-min period of dye using the Alcon Constellation Vision System had no negative impact on cell viability and improved visual acuity by $30 \%$.
\end{abstract}

Correspondence to: Dr Horia Feier, Department of Cardiovascular Surgery, 'Victor Babeş' University of Medicine and Pharmacy Timisoara, 13A Gheorghe Adam Street, 300041 Timisoara, Romania E-mail: horea.feier@gmail.com

Dr Adrian Teodoru, Department of Ophthalmology, 'Lucian Blaga' University of Sibiu, 2A Lucian Blaga Street, 550169 Sibiu, Romania E-mail: ateodoru77@yahoo.com

*Contributed equally

Key words: macular hole, internal limiting membrane, cromovitrectomy, Brilliant Blue G, lutein and zeaxanthin dyes, pars plana vitrectomy, intraocular dyes, staining technique
Staining makes it easier to remove, to be quick and precise while performing macular surgeries. In has been observed that lutein and zeaxanthin dyes offer an intraoperative protective screen that protects photoreceptors more than Brilliant Blue while performing pars plana vitrectomy. Both study groups had good results in time. Surgical visualization is an evolving technology.

\section{Introduction}

Macular hole was first described by Knapp in 1869 and later by Noyes (1872) in patients with ocular trauma $(1,2)$. A macular hole may occur as a result of the development of macular cystoid edema due to inflammation and retinal vascular disease (diabetic retinopathy, vascular occlusions, hypertensive retinopathy, myopia, macular pucker, retinal detachment, and less frequent phototrauma) (3).

The internal limiting membrane (ILM), the basement membrane of the Müller cells, is composed of collagen fibers, glycosaminoglycans, laminin, and fibronectin and it serves as the connection between the vitreous body and the retinal nerve fiber layer. It is said to be almost $1.5 \mu \mathrm{m}$ in the peripheral foveal area, where it is the thickest (4). The ILM helps the cellular proliferation of myofibroblasts, fibrocytes, and retinal pigment epithelium (RPE) cells (5). The role of the ILM is fundamental in the development, structure, and function of the retina, although it can represent a pathologic component, especially in macular holes (6).

Nowadays, surgical techniques such as ILM peeling are widely used for macular hole, macular puckers, epiretinal membranes, diabetic macular edema, retinal detachment, retinal vein occlusions, vitreomacular traction, optic pit maculopathy, and Terson syndrome (7). In a pilot study performed in 1989, Kelly and Wendel performed vitrectomy and posterior cortical removal to ease traction on the macula, shedding light on ILM peeling as a possible therapy for the treatment of full-thickness macular holes. Before this occurred, idiopathic 
macular holes were considered to be untreatable (8). Not much longer after the pilot study, in the 1990s, for the treatment of hemorrhagic macular cysts due to Terson syndrome, Morris et al performed ILM peeling (9).

Intraocular surgery uses non-therapeutic agents as intraocular dyes for one or two steps when needed. The use of dyes for these steps is optional, not mandatory, for surgical success. These substances are efficient, and may even have a beneficial effect on the vitreoretinal surgery. Staining these tissues using vital dyes simplifies the surgical procedure. The first to perform intravitreal injection of a dye on animals was Lobeck in 1932 (10). Sodium fluorescein was the first dye used on humans, in order to identify the transparent vitreous during pars plana vitrectomy (PPV) by Abrams and colleagues (11).

Intraocular dyes primarily used for chromovitrectomy include triamcinolone acetonide (TA) for vitreous identification, indocyanine green (ICG), Brilliant Blue for the identification of the ILM and Trypan Blue (TrB) for epiretinal membrane (ERM) identification (12). TA is a synthetic insoluble corticosteroid. In ocular surgery, TA functions like a dye to stain the vitreous, mainly because of crystal deposition $(13,14)$.

Due to the iodide component and affinity for RPE, ICG introduced by Kadonoso et al is toxic and induces chemical trauma (15).

TrB colors the cell membrane of dead tissues and has a strong affinity for the ERM. Brilliant blue G (BBG) (16) is intense when staining ILM, is easy to remove, and is unique among currently popular dyes. Recently, a good safety profile has also been described for the use of lutein and zeaxanthin-based dyes during ocular surgery.

There are two known techniques for staining the vitreous cavity with vital dyes. The first one is the 'dry technique', when the eye is full of air after removing the liquid. The second one is the 'wet method', when injecting the dye with the eye full of liquid, taking into account the fact that the dye concentration should be lower because it is diluted in the vitreous cavity fluid (17).

Due to the multifactorial etiology of macular holes and in order to fulfill the inclusion and exclusion criteria, all subjects enrolled in this study were tested for associated ocular and general diseases. All patients enrolled in this study underwent ophthalmological evaluation that included: Visual acuity, intraocular pressure, anterior pole and posterior pole examination, in order to establish the macular hole etiology and any surgery contraindications. Also, a complete blood count was performed during hospitalization (18-25).

The aim of this study was to present and investigate the safety and efficacy of vital dyes for macular pathology after PPV in large full-thickness macular holes (MHs). The design of the study was constituted by comparative retrospective case series.

\section{Patients and methods}

Patients. Thirty eyes of 30 different patients with MHs were utilized in the study. The cause of the visual acuity drop was an idiopathic MH. We divided the patients into two groups, depending on the dye used intraoperatively: 15 eyes colored with Brilliant blue $\mathrm{G}$ (BBG group) and 15 eyes colored with lutein/zeaxanthin (L/Z group).

The study was approved by the Ethics Committee (number 403) of 'Dr. Carol Davila' Central Military Emergency University Hospital Bucharest. Informed consent was signed by all patients. Patients inclusion and exclusion criteria are presented in Table I.

Surgery. Surgery was conducted under retrobulbar anesthesia, with a 3-port 25-gauge vitrectomy, using the Alcon CONSTELLATION $^{\circledR}$ Vision System (Alcon Romania S.R.L.). After core vitrectomy, intravitreal TA was injected to stain the posterior cortical vitreous, and the ILM was stained with $0.25 \mathrm{mg} / \mathrm{ml}$ BBG solution or L/Z dyes. The inverted ILM flap technique was applied using the inherent elastic properties of the retina. The graft was held with Grieshaber ${ }^{\mathrm{TM}}$ end-grasping forceps (Alcon Romania S.R.L.) to bring the edges of the macular hole closer. Fluid-gas exchange was then performed. No intraoperative complications were encountered. Postoperatively, the patient was postured supine for one week, and the retina was attached with no postoperative complications.

Assessment. We reviewed and collected data in regards to: IOP (after 1 month), the Watzke-Allen test, visual acuity (VA) and optical coherence tomography (OCT) parameters, six months after the vitrectomy surgery with standard ILM peeling using BBG and L/Z dyes. The VA measurements were performed using the Snellen method. OCT changes were judged by a single retinal specialist. There are 2 types of macular hole closure based on OCT: Type 1 (closed without retinal neurosensory foveal defect) and type 2 (closed with foveal neurosensory retinal defect). The magnitude of the postoperative visual improvement of type 1 closure was greater than that of type 2 closure. Allen test was used in the diagnosis of a macular hole in the retina. Over the macula, a thin line of slit lamp light is projected and the patient is asked to report on its appearance. A line appearing broken may indicate a macular hole. A line appearing deformed suggests epiretinal membrane. A line that seems to be thin indicates macular edema or incipient macular hole (stage I, II).

Statistical analysis. For the data systematization, we used the Excel program of the Microsoft Office 365 suite. Graphical representations and statistical analysis of the data were performed using the ' $\mathrm{R}$ ' program, 3.0.1 version.

\section{Results}

The distribution of the patients by age group and sex are shown (Figs. 1 and 2). We observed that the most represented age group was from 65 to 70 years. Gender impact is to be considered, as females are more likely to develop a MH.

Average VA before surgery for the BBG group was 0.18 and for the $\mathrm{L} / \mathrm{Z}$ group was 0.15 . After surgery, the mean VA for the BBG group was 0.45 and for the $L / Z$ group was 0.42 (Figs. 3 and 4).

For the entire study lot, the mean VA after surgery increased by $30 \%$. The VA improvement has a positive impact on the patient quality of life. By comparing the two groups, VA improvement was relatively similar. 
Table I. Patient inclusion and exclusion criteria.

Inclusion criteria

Exclusion criteria

Patients enrolled at the Department of Ophthalmology,

'Dr. Carol Davila' Central Military University Emergency Hospital, Bucharest

Large full-thickness idiopathic $\mathrm{MH}$ (>400 microns)

Minimum follow-up period of 6 months

$\mathrm{VA}>0.1$
Significant epiretinal membrane

Macular pathology (neovascular age-related macular degeneration, foveal involving geographic atrophy)

Retinal pathology (retinal laser, retinal vascular occlusion, diabetic retinopathy)

Optic nerve pathology (congenital anomalies, tumors, glaucoma) Previous intraocular injection

MH, macular hole; VA, visual acuity.

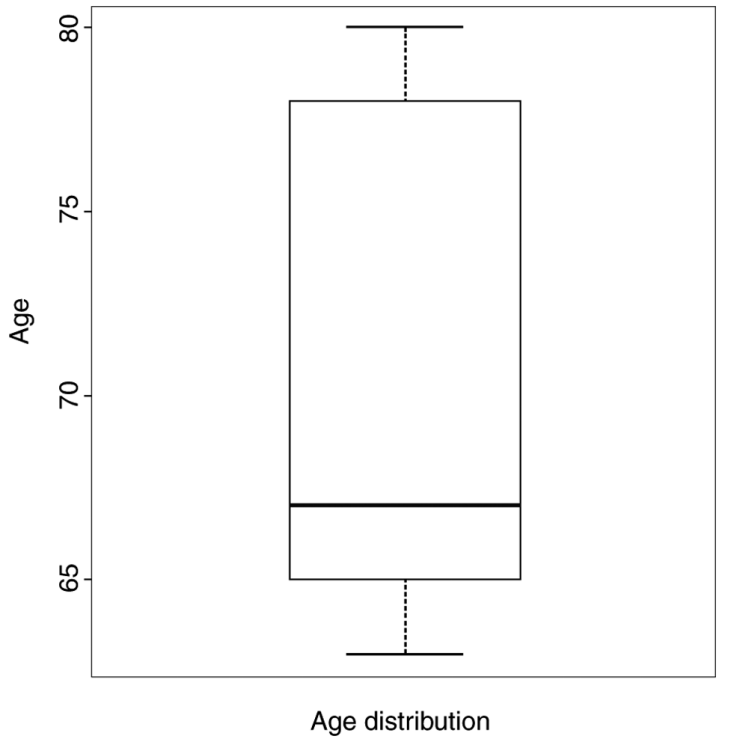

Figure 1. Average age (years) of the entire study group.

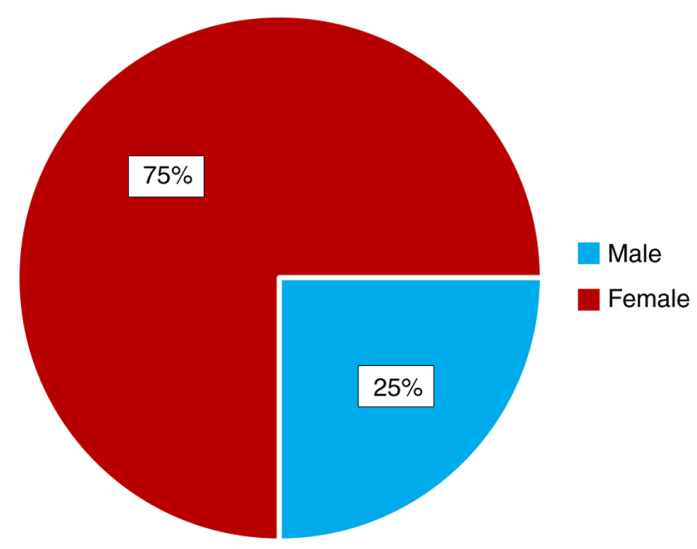

Figure 2. Sex distribution of the entire study group.

This improvement takes into account numerous factors that involve conditions related to the surgery and other associated conditions, such as preoperative lens opacification, degree of post-op opacification, MH etiology, and patient compliance.

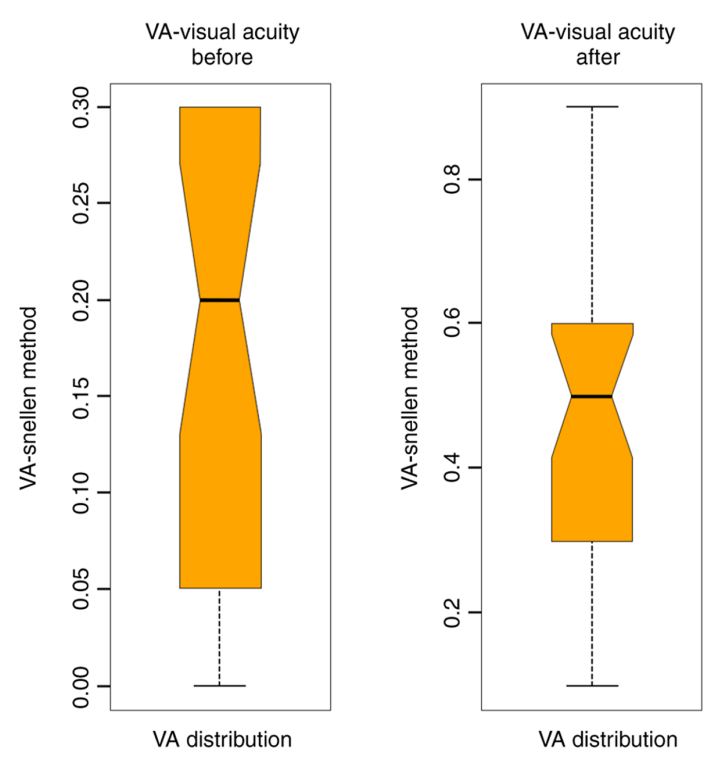

Figure 3. Improvement in visual acuity (VA) 6 months after surgery.
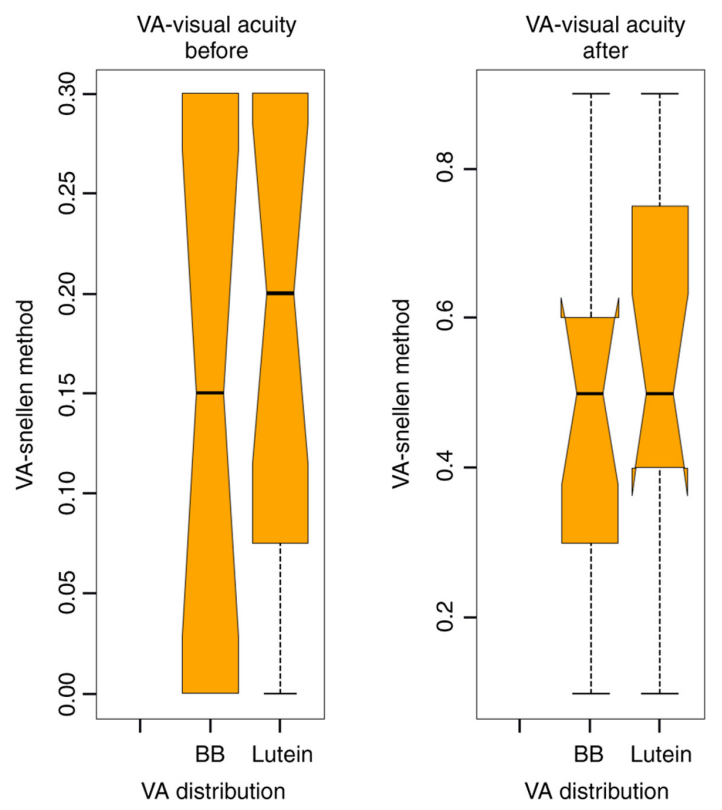

Figure 4. Evolution of visual acuity (VA) according to the dye used. 

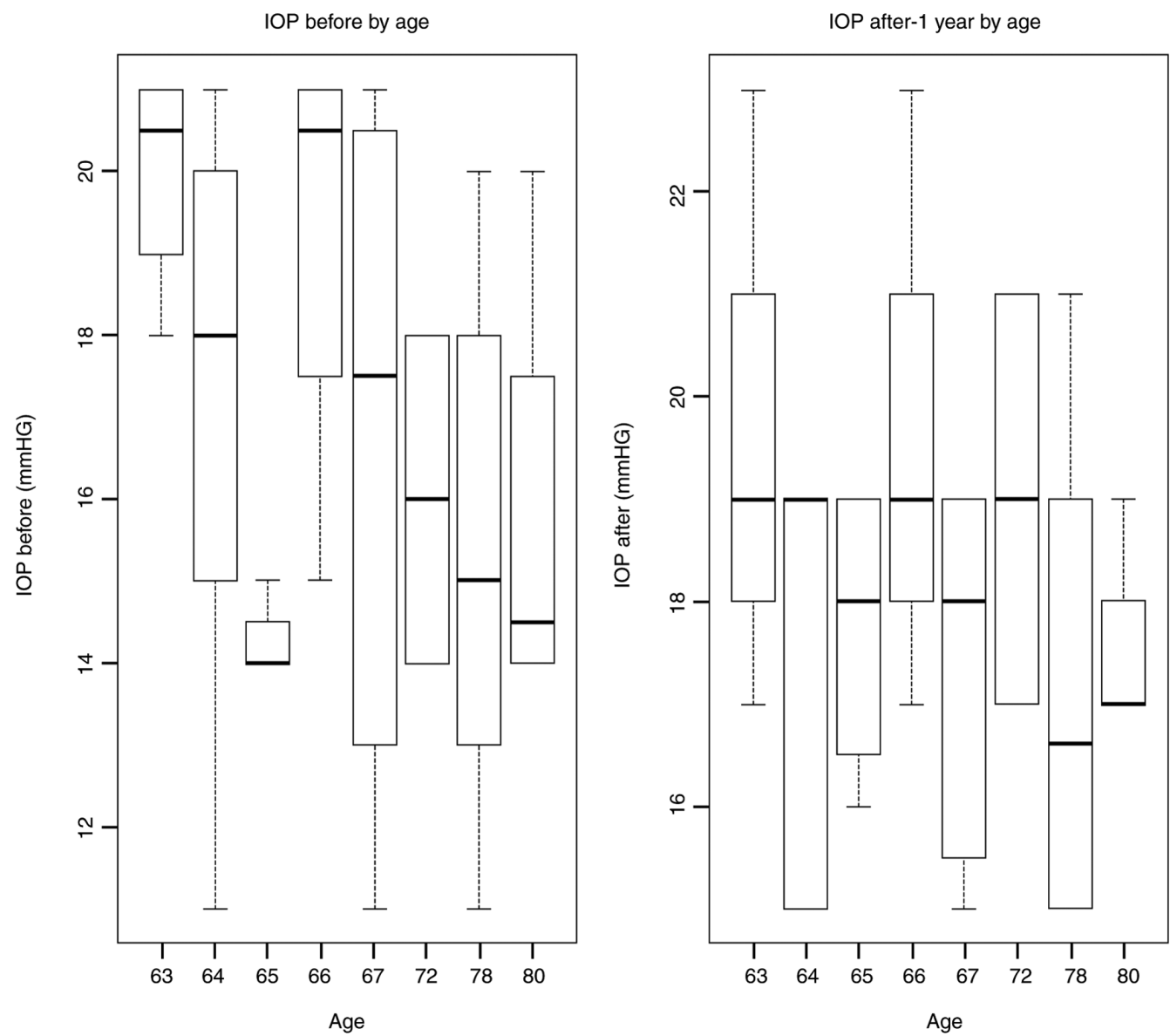

Figure 5. IOP by age (years), at one month after surgery.

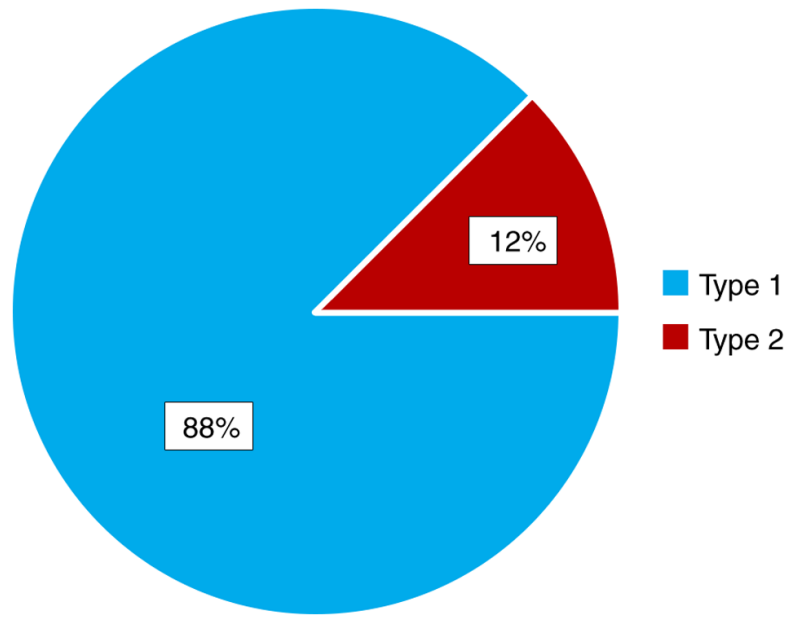

Figure 6. OCT closing type. OCT, optical coherence tomography.

The average IOP before surgery was $16 \mathrm{mmHg}$; after surgery ( 1 month) it was $18.5 \mathrm{mmHg}$. This asymmetry comes from the fact that TA was used to highlight the vitreous gel. TA persists in the eyes for about 9-12 months. Increases in IOP being recorded mainly in the first days-4 weeks. Later, due to the dilution of TA, IOP increases are less frequent.

Patients did not follow glaucomatous treatment before and after surgery as long as this pathology fell into the exclusion criteria (Fig. 5).

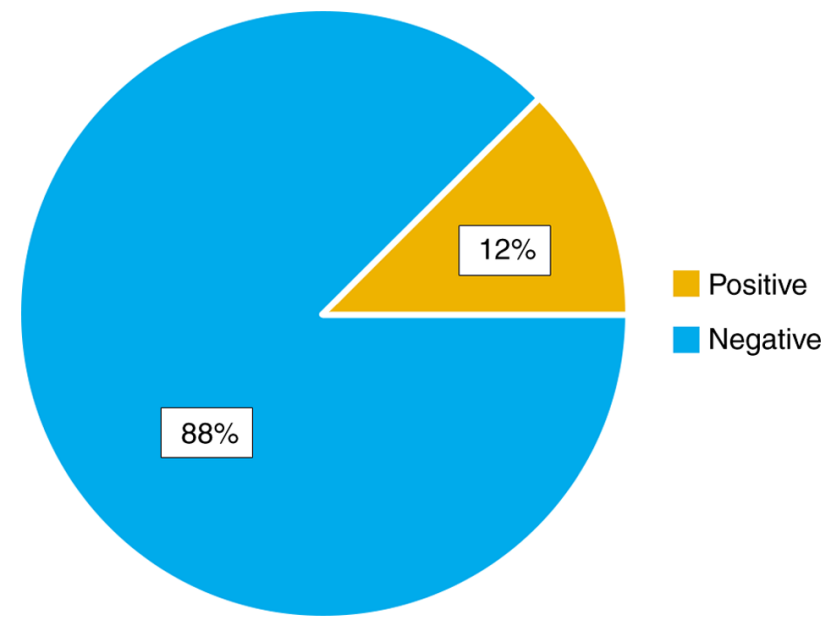

Figure 7. Watzke Allen Test results.

We evaluated the $\mathrm{MH}$ closure rate and we noted that it was more than $85 \%$ type 1 in both studied groups, without statistical differences between them (Fig. 6).

In our case series, we observed that VA improvement occurred in the first month after pars plana vitrectomy.

The typical manifestation that consisted of metamorphopsia, central scotoma was remedied postoperatively. The Watzke-Allen test was negative in a high percentage after surgery (Fig. 7). 
$\mathrm{BB}$, lutein and zeaxanthin dyes color the target tissue offering safety issue and prospects.

\section{Discussion}

In 2012, 102 eyes were analyzed after lutein dye solution alone or combined with Brilliant blue (BB) or TrB was injected directly over the different intraocular structures and improved the ability to initiate peeling in cadaveric eyes, with no clinical or histologic signs of toxicity (26). In addition, in 2012, 60 cadaveric eyes were stained along with lutein and zeaxanthin dye or in combination with different Brilliant Blue concentrations and efficiently stained vitreous and internal limiting membrane, with no dye solutions in the eyes after the membrane removal (27).

Badaro et al reported that a combination of soluble lutein/zeaxanthin (LZ) $1 \%$ and Brilliant blue (BB) $0.025 \%$, facilitated surgical steps and showed no signs of toxicity at 1 month of follow-up in 18 eyes treated surgically for a macular hole (MH) or epiretinal membranes (ERM) (28).

Casaroli-Marano et al demonstrated that L/Z-based dye solutions, either alone or in association with $\mathrm{BB}$ or $\operatorname{TrB}$ did not significantly alter mitochondrial activity in the cell lines tested; in addition, no structural alterations were observed in the neurosensory retina, retinal pigment epithelium (RPE), or choriocapillaris-choroidal complex (29).

In 2014, Maia et al evaluated 12 eyes that underwent surgery using lutein-based dye. They histologically examined the peeled membranes and claimed successful intraoperative identification (30).

A crystalline lutein-based dye called Vitreodyne ${ }^{\mathrm{TM}}$ proved to be superior to the existing alternative dyes after staining 18 patients with a diagnosis of ERM and MHs (31).

In the present study, we used BB dye for the first group and $\mathrm{L} / \mathrm{Z}$ dyes for the second group, and our results confirmed that both dyes are useful intraocular tools in order to obtain good surgical results.

The study had its limitations, because of the reduced number of study eyes and because of the lack of electrophysiology studies.

In conclusion, optical coherence tomography (OCT), the gold standard diagnostic tool for retinal diseases, is extremely useful for preoperative evaluation of MHs and postoperative surgical results. MH size is typically predictive of postoperative outcomes.

Identifying the internal limiting membrane (ILM) is a challenging step in surgery, since the ILM is a barely visible membrane; identifying and removal of the ILM is difficult even for experienced retinal surgeons. Staining can reduce surgical trauma to the retina during ILM removal, thus ILM staining with vital dyes is essential for increased visibility of the ILM. Coating materials only cover the membrane surface and do not stain the ILM.

Our results indicate that from a clinical, intraoperative point of view, the retinal details can be observed only when the whole vitreous mass has been removed. Adding intraocular dyes increases ILM visualization, offering the surgeon a better and safer surgical approach. In our study, we used two different dyes, but no major differences were noted between the two groups. In all cases, ILM was identified and removed. We may consider that the protective screen formed by intraocular dyes protects the retinal cells from the phototoxic effect.
The association between the inverted ILM flap technique and intraocular dyes offers good results regarding VA and the MH closure rate.

To sum up, our study confirmed that both the BBG and $\mathrm{L} / \mathrm{Z}$ groups of eyes exhibited in our retrospective study, were equivalent in mean age affected with full-thickness MHs, exhibited improved VA at 6-month after surgery confirming the fact that intraocular dyes facilitate the surgical steps and have no toxic effect on the retinal cells.

\section{Acknowledgements}

Professional editing, linguistic and technical assistance were performed by Irina Radu, Individual Service Provider.

\section{Funding}

No funding was received.

\section{Availability of data and materials}

All data and materials supporting the results of the present study are available in the published article.

\section{Authors' contributions}

ISP designed the study and was responsible for the acquisition and interpretation of the data. SN provided scientific advice. $\mathrm{OM}, \mathrm{CCC}$, AT and $\mathrm{CD}$, were involved in the design of the study; they carefully inspected and also revised the manuscript. SS, CP and HF were involved in the conception and drafting of the study and revised the manuscript. All authors read and approved the final manuscript.

\section{Ethics approval and consent to participate}

The study was approved by the Local Ethics Committee of Dr. Carol Davila' Central Military Emergency University Hospital Bucharest (no. 403). Informed consent was signed by all patients.

\section{Patient consent for publication}

Not applicable.

\section{Competing interests}

The authors declare that they have no competing interests.

\section{Authors' information}

The first author, Ioana Stella (Patoni) Popescu, is a PhD student at the Department of Ophthalmology of the 'Victor Babes' University of Medicine and Pharmacy in Timisoara where she is solely pursuing her $\mathrm{PhD}$ thesis.

\section{References}

1. Knapp H: About isolated ruptures of the choroid as a result of trauma to the eyeball. Arch Augenheilkd 1: 6-29, 1869 (In German).

2. Noyes HD: Detachment of the retina with laceration at the macula. Trans Am Ophthalmol Soc 1: 128-129, 1871. 
3. Bikbova G, Oshitari T, Baba T, Yamamoto S and Mori K Pathogenesis and management of macular hole: Review of current advances. J Ophthalmol 2019: 3467381, 2019.

4. Christensen UC: Value of internal limiting membrane peeling in surgery for idiopathic macular hole and the correlation between function and retinal morphology. Acta Ophthalmol 87: 1-23, 2009.

5. Almony A, Nudleman E, Shah GK, Blinder KJ, Eliott DB, Mittra RA and Tewari A: Techniques, rationale, and outcomes of internal limiting membrane peeling. Retina 32: 877-891, 2012

6. Gelman R, Stevenson W, Prospero Ponce C, Agarwal D and Byron Christoforidis JB: Retinal damage induced by internal limiting membrane removal. J Ophthalmol 2015: 939748, 2015.

7. Walia HS, Shah GK and Hariprasad SM: ILM peeling a vital intervention for many vitreoretinal disorders. Ophthalmic Surg Lasers Imaging Retina 45: 92-96, 2014

8. Kelly NE and Wendel RT: Vitreous surgery for idiopathic macular holes: Results of a pilot study. Arch Ophthalmol 109: 654-659, 1991.

9. Morris R, Kuhn F and Witherspoon CD: Hemorrhagic macular cysts. Ophthalmology 101: 1, 1994.

10. Lobeck E: Investigations into the role of retinal tear in skin detachment. Experimental investigations on the intraocular fluid change in artificial netskin detachment. Alb v. Graefes Arch Ophthalmol 128: 513-573, 1932 (In German).

11. Abrams GW, Topping T and Machemer R: An improved method for practice vitrectomy. Arch Ophthalmol 96: 521-525, 1978.

12. Farah ME, Maia M and Rodrigues EB: Dyes in ocular surgery: Principles for use in chromovitrectomy. Am J Ophthalmol 148: 332-340, 2009.

13. Dubey AK: Trypan blue enhanced vitrectomy in clear gel vitrectomy. Indian J Ophthalmol 51: 286-287, 2003.

14. Peyman GA, Cheema R, Conway MD and Fang T: Triamcinolone acetonide as an aid to visualization of the vitreous and thre posteror hyaloid during pars plana vitrectomy. Retina 20: 554-555, 2000.

15. Neuman GO, Holbach H and Kruse FE: Applied Pathology for Ophthalmic Microsurgens. 5.6 Retina and Vitreous. Springer, pp300-301, 2008.

16. Totan Y, Güler E and Dervişoğulları MS: Brilliant blue G assisted epiretinal membrane surgery. Sci Rep 4: 3956, 2014.

17. Musat O, Stefan C, Boariu AM, Colta D, Cernat C, Alexandru L, Georgescu RD, Patoni IS, Timaru CM and De Algerino S: Chromovitrectomy. Rom J Ophthalmol 60: 59-62, 2016.

18. Preda MA, Popa G, Karancsi OL, Musat O, Popescu SI, Munteanu M and Popa Z: Effectiveness of subconjunctival bevacizumab associated with a laser-based procedure in the treatment of neovascular glaucoma. Farmacia 66: 621-626, 2018.

19. Boruga O, Bălăşoiu AT, Giuri S, Munteanu M, Stanca HT, Iovănescu $G$ and Preda MA: Caruncular late-onset junctional nevus: Apropos of an anatomo-clinical observation. Rom J Morphol Embryol 58: 1461-1464, 2017.

20. Balica NC, Poenaru M, Preda MA, Boia RE, Burlacu ON, Horhat ID, Mogoanță CA, Vlăescu AN, Baderca F, Jifcu EM and Sarău CA: Primary tonsillar tuberculosis-case report. Rom J Morphol Embryol 60: 267-271, 2019.
21. Popa G, Karancsi OL, Preda MA, Suta MC, Stelea L, Musat O, Popescu SI, Balica NC, Bogdanici C and Munteanu M: Assessment of Pain during laser-based procedures in the treatment of glaucoma. Rev Chim 70: 2105-2107, 2019.

22. Preda MA, Karancsi OL, Munteanu M and Stanca HT: Clinical outcomes of micropulse transscleral cyclophotocoagulation in refractory glaucoma-18 months follow-up. Lasers Med Sci 35: $1487-1491,2020$

23. Stanca HT, Munteanu M, Jianu DC, Motoc AGM, Tăbăcaru B, Stanca S, Ungureanu E, Boruga VM and Preda MA: New perspectives in the use of laser diode transscleral cyclophotocoagulation. A prospective single center observational cohort study. Rom J Morphol Embryol 59: 869-872, 2018.

24. Stanca HT, Munteanu M, Jianu DC, Motoc AGM, Jecan CR, Tăbăcaru B, Stanca S and Preda MA: Femtosecond-LASIK outcomes using the VisuMax ${ }^{\circledR}-$ MEL $^{\circledR} 80$ platform for mixed astigmatism refractive surgery, Rom J Morphol Embryol 59: 277-283, 2018.

25. Munteanu M, Rosca C and Stanca HT: Sub-inner limiting membrane hemorrhage in a patient with Terson syndrome. Int Ophthalmol 39: 461-464, 2019.

26. Brandão-Lencart J and Sousa-Martins D: New lutein-based dyes for ophthalmic surgery use. Kemin Inspired Molecular Solutions 2012. http://peschkemed.com/wp-content/uploads/2013/10/ Retina-.pdf.

27. Sousa-Martins D, Maia M, Moraes M, Lima-Filho AA, Rodrigues EB, Chen J, Farah ME, Santos LB and Belfort R Jr: Use of lutein and zeaxanthin alone or combined with Brilliant Blue to identify intraocular structures intraoperatively. Retina 32: 1328-1336, 2012

28. Badaro E, Furlani B, Prazeres J, Maia M, Alves Souza Lima A, Souza-Martins D, Muccioli C, Adami Lucatto LF and Belfort R Jr: Soluble lutein in combination with brilliant blue as a new dye for chromovitrectomy. Graefes Arch Clin Exp Ophthalmol 252: 1071-1078, 2014.

29. Casaroli-Marano RP, Sousa-Martins D, Martínez-Conesa EM, Badaró E, Nunes RP, Lima-Filho AA, Rodrigues EB, Belfort R Jr and Maia M: Dye solutions based on lutein and zeaxanthin: In vitro and in vivo analysis of ocular toxicity profiles. Curr Eye Res 40: 707-718, 2014.

30. Maia M, Furlani BA, Souza-Lima AA, Martins DS, Navarro RM and Belfort R Jr: Lutein: A new dye for chromovitrectomy. Retina 34: 262-272, 2014

31. Singh P, Deuchler S, Mueller M, Kohnen T, Sousa-Martins D, Pinheiro-Torres B and Koch FHJ: Chromovitrectomy with vitreodyne $^{\mathrm{TM}}$. Invest Ophthalmol Vis Sci 57: 4452, 2016. 